\begin{tabular}{|c|c|}
\hline Title & Mechanical Effect of A cetic A cid Lignin A dsorption on Honeycomb-Patterned Cellulosic Films \\
\hline Author(s) & $\begin{array}{l}\text { Uraki, Y asumitsu; Matsumoto, Chinatsu; Hirai, T akuro; T amai, Y utaka; Enoki, Makiko; Y abu, Hiroshi; Tanaka, } \\
\text { Masaru; Shimomura, Masatsugu }\end{array}$ \\
\hline Citation & $\begin{array}{l}\text { Journal of Wood Chemistry and Technology, 30(4), 348-359 } \\
\text { https://doi.org/10.1080/02773811003746709 }\end{array}$ \\
\hline Issue Date & $2010-10$ \\
\hline Doc URL & http:/hdl.handle.net/2115/47192 \\
\hline Rights & $\begin{array}{l}\text { This is an electronic version of an article published in Journal of Wood Chemistry and Technology, 30(4), 2010, Pages } \\
\text { 348-359. Journal of Wood Chemistry and Technology is available online at: } \\
\text { http://www.informaworld.com/openurl?genre=article\& issn=0277-3813\&volume=30\& issue=4\& spage=348 }\end{array}$ \\
\hline Type & article (author version) \\
\hline File Information & JWCT30-4_348-359.pdf \\
\hline
\end{tabular}

Instructions for use 


\section{Mechanical effect of acetic acid lignin adsorption on honeycomb-patterned cellulosic}

\section{films}

Yasumitsu Uraki ${ }^{*}$, Chinatsu Matsumoto ${ }^{2}$, Takuro Hirai ${ }^{1}$, Yutaka Tamai ${ }^{1}$, Makiko Enoki $^{3}$, Hiroshi Yabu ${ }^{4}$, Masaru Tanaka ${ }^{4}$, Masatsugu Shimomura ${ }^{4}$

${ }^{1}$ Research Faculty of Agriculture, Hokkaido University, Sapporo 060-8589, Japan

${ }^{2}$ Graduate School of Life Science, Hokkaido University, Sapporo 060-0810, Japan

${ }^{3}$ Department of Ocean Science, Tokyo University of Marine Science and Technology, Tokyo 108-8477, Japan

${ }^{4}$ Institute of Interdisciplinary Research for Advanced Materials, Tohoku University Sendai, 980-8577, Japan.

\section{ABSTRACT}

We have already fabricated honeycomb-patterned cellulosic films with cellulose I and II polymorphisms as a basal framework in order to create an artificial woody cell wall ${ }^{[1,2]}$. The adsorption of an isolated lignin, acetic acid lignin (AL), was attempted onto the honeycomb films not only to develop materials further mimicking the cell wall but also to elucidate the mechanical effect of isolated lignin on the tensile strength of the cellulosic architecture. The tensile strengths of honeycomb-patterned cellulosic films were improved by the AL adsorption. Although the cellulosic films without lignin weakened under high moisture content conditions as compared with those under the low content conditions, the lignin-adsorbed cellulosic film maintained significant tensile strength even under the high content conditions. This result suggests that lignin contributes to reinforce the mechanical strength of cellulose framework, in particular high moisture conditions.

Keywords; Artificial woody cell wall, Honeycomb-patterned cellulosic film,

Lignin adsorption, Tensile strength

\footnotetext{
*Author for correspondence. E-mail: uraki@for.agr.hokudai.ac.jp
} 


\section{INTRODUCTION}

A woody cell wall is constructed by hierarchical deposition of the essential cell wall components, cellulose, hemicelluloses and lignin, on the plasma membrane, ${ }^{[3,4]}$ which results in the formation of a honeycomb like array. In this constructional array, cellulose, together with the other components maintains wood morphology and resists external mechanical stress. The actual function of lignin and hemicelluloses on the mechanical properties of the woody cell wall architecture, however, has not yet been clearly elucidated. We made a strategy to elucidate the functions of such the components by using cell wall-mimicking materials, which we term as "artificial woody cell wall”.

We have developed honeycomb-patterned cellulosic films with cellulose I and II polymorphisms, which had uniform pores. ${ }^{[1,2]}$ The honeycomb-patterned films with cellulose II polymorphism were easily prepared by the combination of self-organization process of amphiphilic polymer and the transcription method with polydimethyl siloxane (PDMS) ${ }^{[1]}$ In the former process, a convex honeycomb-patterned film template with uniform pore size was first fabricated by blowing humid air onto the polymer solution. In the second step, transcription process, PDMS together with a curing agent was poured onto the convex template film to form a second concave template film that had an opposite morphology of first one. The second template was finally stamped onto a chloroform solution of cellulose triacetate followed by deacetylation to give a honeycomb-patterned film with cellulose II polymorphism. Similarly, the fabrication of a honeycomb film with cellulose I polymorphism was produced by controlling the movement of a cellulose-producing bacteria, Gluconacetobacter xylinus, which was cultured on a concave honeycomb-patterned agarose gel surface under high humidity and $\mathrm{CO}_{2}$ concentration. ${ }^{[2]}$

Analogous to other honeycomb-patterned materials ${ }^{[5-7]}$ these cellulosic films may provide a novel use of cellulose as a functional material. In addition, because of their hexagonal array morphology these honeycomb-patterned cellulosic films can potentially serve as a basal framework for fabricating artificial woody cell walls. Therefore, it is a potential model to experimentally elucidate the deformation mechanisms of woody cell walls, particularly as its morphology is very similar to a proposed model used for the clarification of cell wall deformation. ${ }^{[8,9]}$ Furthermore, it will be a useful model to elucidate the function of cell wall components after deposition of hemicellulose and lignin onto the honeycomb-patterned cellulosic films.

In this study, lignin-adsorbed honeycomb-patterned cellulosic films with cellulose I and II polymorphisms were prepared, and used to investigate the influence of isolated lignin on the physical properties of cellulosic cell wall architectures. Acetic acid lignin (AL) derived by atmospheric acetic acid pulping was used as an isolated lignin. It has been shown to act as a 
hot-melt type adhesive, reinforcing the mechanical strength of cellulosic fibers in fiberboard-like cellulosic composites and giving them water repellency, ${ }^{[10,11]}$ similar properties to those proposed for native lignin in the tree. The tensile properties of the materials produced were measured on a testing apparatus designed for this study, and the measurements were conducted under two conditions, high and low moisture content to assess the water repellency of the adsorbed lignin.

\section{EXPERIMENTAL}

\section{Fabrication of templates}

A mixture (10:1) of poly( $\varepsilon$-caprolactone) and copolymer (dodecylacrylamide and $\omega$-carboxyhexylacryl-amide) ${ }^{[12]}$ was dissolved in chloroform to yield a $2 \mathrm{mg} / \mathrm{mL}$ solution. Humid air was blown onto the solution $(10 \mathrm{~mL})$ using a simple apparatus as shown in Figure 1. After evaporation of the solvent, a convex (hexagonal mountain range) honeycomb-patterned film with uniform pores of $25 \mu \mathrm{m}$ in diameter was produced as the first template. Honeycomb-patterned films with different pore sizes were fabricated from various volumes of the polymeric solutions.

A viscous liquid of polydimethylsiloxane (PDMS) with curing agents (SYLGARD 184 Silicone Elastomer Kit, Dow Corning Co. Midland, MI) was poured onto the first template and cured at room temperature for $48 \mathrm{~h}$. The resultant elastomer was then peeled off to yield the second template. Similarly, a third template was prepared by the transcription of the second template with PDMS. ${ }^{[1,2]}$

\section{Preparation of cellulosic honeycomb films}

The second template was stamped onto a chloroform solution $(2 \mathrm{mg} / \mathrm{mL}$ ) of cellulose triacetate (CTA). After evaporation of the solvent the template was peeling off, and a honeycomb-patterned CTA film was obtained. A convex honeycomb-patterned cellulosic film with cellulose II polymorphism was prepared by deacetylation of the CTA film with $\mathrm{NaOCH}_{3} / \mathrm{CH}_{3} \mathrm{OH}$ at room temperature over night.

A convex honeycomb-patterned film with cellulose I polymorphism was fabricated by culturing G. xylinus (ATCC 53582) in a high carbon dioxide concentration (90\%) atmosphere on a concave honeycomb-patterned agarose film with Hestrin-Schramm medium, which was prepared by pressing the third template onto a hot agarose aqueous solution. ${ }^{[2]}$

The micrographs and electron diffraction diagrams of the resultant honeycomb-patterned films were taken with a JEOL-2000EX II TEM operated at $100 \mathrm{kV}$, and recorded on 
Mitsubishi MEM film. Low dose defocused imaging in the bright field mode was used to visualize the sample without further contrast enhancement. The images were taken at 2500 6000x magnification. FT-IR spectra of the specimens on the copper grid of TEM were measured on a Perkin Elmer Spectrum One equipped with a microscope attachment. Spectra were recorded by an MCT detector, accumulating 128 scan from $4000-700 \mathrm{~cm}^{-1}$ with a resolution of $4 \mathrm{~cm}^{-1}$. The aperture was $50 \mu \mathrm{m} \times 50 \mu \mathrm{m} .^{[2]}$

\section{Microscopic observation of bacterial movement on an agarose film with honeycomb-patterned grooves}

An agarose film with honeycomb-patterned grooves was placed in a chamber equipped with a gas inlet and outlet tubes, glass window, and heat controller. An aliquot of bacterial medium preincubated for half a day was then dropped onto the film, and the air in the chamber was exchanged with $90 \% \mathrm{CO}_{2}$, and the chamber was sealed. The time course of bacterial incubation was observed on a real-time confocal microscope (C9100-13, Nikon) in the Nikon Image Center of Hokkaido University. The images were taken every $10 \mathrm{~s}$ for 700 ms of exposure time. The time-lapse images were converted to movie by using AQUACOSMOS Ratio Imaging software. Other optical microscopic images were taken using a 3-D laser microscope, VK-9500, KEYENCE, Osaka, Japan.

\section{Deposition of isolated lignin onto honeycomb films and bromination of deposited lignin}

Acetic acid lignin (AL) was dissolved in $1 \mathrm{M} \mathrm{NaOH}$ aqueous solution to give a $5 \%(\mathrm{w} / \mathrm{v})$ solution. The cellulosic films were immersed into the solution and left overnight at room temperature. The films were then washed thoroughly with distilled water until the filtrate was a neutral $\mathrm{pH}$, and subsequently dried in vacuo at $40{ }^{\circ} \mathrm{C}$.

The films were placed in a vessel containing liquid bromine in a beaker, which was filled with gaseous bromine. ${ }^{[13]}$ The bromine location in the film was analyzed by the scanning electron microscope equipped with energy dispersive X-ray spectrometer (SEM-EDX).

\section{Measurement of tensile strength}

A testing apparatus was specially designed to measure the tensile properties of small thin honeycomb specimens as shown in Figure 2. In a typical test, a specimen was fixed to a paper sample holder with an epoxy adhesive, and the holder was then fixed to the support base using small bolts, nuts and rectangular washers. The paper sample holder was then cut to make the specimen free supporting, and it was forced to elongate at a low strain rate by 
turning the grip of the micrometer shown in Figure 2. In this test, the elongation and responding tensile load were monitored synchronously by a linear variable displacement transducer of $\sim 1 / 1000 \mathrm{~mm}$ minimum resolution and a load cell of $100 \mathrm{~g}$ capacity and $0.05 \mathrm{~g}$ minimum resolution, respectively. The measurements were repeated at least three times (normally five times) for each specimen under a low moisture content atmosphere $\left(22^{\circ} \mathrm{C}\right.$, relative humidity (RH) of $50 \%)$ and high moisture atmosphere $\left(30^{\circ} \mathrm{C}, \mathrm{RH} 80 \%\right)$ in an air-conditioned chamber. Modulus of elasticity (MOE) was calculated from initial displacement and load, and tensile strength and elongation were calculated from the load and elongated length ratio to original specimen length at failure.

\section{RESULTS AND DISCUSSION}

\section{Confirmation of bacterial movement along the hexagonal microgrooves to fabricate honeycomb-patterned film with cellulose I polymorphism}

Honeycomb-patterned films with cellulose I polymorphism were fabricated by culturing $G$. xylinus on an agarose medium with a concave honeycomb surface (array of hexagonal grooves) as shown in Figure 3 (A and B). Although Figures 3C and 3D show the resultant honeycomb-patterned cellulose I films, they do not show direct evidence of the path taken by the bacterium along the array of hexagonal grooves. This is important, as the track of bacterial movement would affect the assembled structure of the cellulosic microfibrils. To observe the bacterial motion on the hexagonal microgroove film, the bacterium was cultured in a special chamber, and time-lapse images every $10 \mathrm{~s}$ were taken using a real-time confocal microscope.

Figure 4 shows the bacterial motion on the surface of the agarose film with the honeycomb pattern microgrooves (a video of this is available in reference 14). It can be seen that some single body bacteria moved rotationally along the hexagonal grooves, while long bacteria, which consist of a few bodies and might do the cell division, migrated transversally along several hexagonal grooves. Most of the bacteria moved in an anticlockwise direction, except for one bacterium that moved in a clockwise direction. The mobile speed was approximately $3 \mu \mathrm{m} / \mathrm{min}$. It was deduced from the tracks of bacterial motion that the structure of honeycomb-patterned bacterial cellulose was produced by cylindrical microfibril deposition connected by transverse microfibrils.

The crystalline structures of the obtained honeycomb-patterned films were analyzed by electron diffraction and FT-IR spectroscopy. The honeycomb-patterned bacterial cellulose indicated a highly ordered cellulose $\mathrm{I} \alpha{ }^{[2]}$ while a cellulose II polymorphism was confirmed for the honeycomb-patterned film prepared from CTA. However, because of the film thinness we were not able to measure the crystallinities of the two films by X-ray diffraction. 


\section{Deposition of AL onto honeycomb-patterned film}

An isolated lignin, AL, was adsorbed onto the honeycomb-patterned cellulosic films to further mimic the woody cell wall structure. Figure 5 shows SEM-EDX images and bromine distribution in a honeycomb-patterned film and a flat film without significant pore structure. It is well known that lignin reacts with bromine, therefore, lignin bromination is widely used for labeling lignin. ${ }^{[13]}$ As expected, bromine was located only on the surface of the films. This lignin distribution seems to be very similar to that of a wood cell wall, where lignin concentration is higher in the cell corner and middle lamella than in the secondary wall or cellulosic matrix.

The adsorbed amount of lignin in the honeycomb-patterned film with cellulose I polymorphism was determined to be about $32 \%$ by the Klason method. Interestingly, this value is similar to the content of lignin in softwoods.

\section{Relationship between honeycomb pore size and tensile properties}

In the present study it was very difficult to control the pore size in the fabrication of the honeycomb-patterned films with cellulose I polymorphism because of the restriction imposed by the bacterial body size. On the other hand, pore size was easily controlled in the film fabrication with cellulose II polymorphism because we were able to control the pore size of the first template by varying the polymer solution volume and concentration. Thus, we prepared several sheets of honeycomb film with cellulose II polymorphism to estimate the effects of pore size and thickness of fabricated films on tensile properties.

Figure 6 shows the modulus of elasticity and tensile strength versus honeycomb pore size or film thickness. The correlation coefficients (R) between MOE and pore size of the honeycomb-patterned regenerated cellulosic film (cellulose II) were -0.63 and -0.82 for the films without and with lignin adsorption, respectively, and the $\mathrm{R}$ between tensile strengths and pore size were -0.75 and -0.66 , respectively. The R between MOE or tensile strength and film thickness, on the other hand, were -0.30 and 0.17 or -0.52 and 0.22 for the corresponding specimens. These results show a clear negative correlation between the pore size and the tensile properties of the honeycomb-patterned films, which suggest a dependence of the tensile properties on the actual cellulose ratios determined by the pore size in the cross sectional areas of the films. On the other hand, film thickness had reasonably little effect because the cellulose volume content was considered to be constant throughout the film thickness.

Table 1 summarizes tensile properties of several cellulosic films tested in this study 
except those of the honeycomb-patterned films with cellulose II polymorphism shown in Figure 6. The tensile properties of the honeycomb-patterned films with cellulose I polymorphism were much lower than those estimated simply from the average volume ratio, $71 \%$ of cellulose in the honeycomb-patterned films. This result indicates two dimensional structural behavior in in-plane mechanical response of the honeycomb-patterned cellulosic framework, which might simulate the mechanical characteristics of wood in radial-tangential plane or perpendicular to the grain that are generally from $1 / 5$ to $1 / 30$ of those parallel to the grain.

\section{Effect of lignin deposition onto honeycomb-patterned film on tensile properties}

The tensile strength was measured under two conditions, high and low moisture content atmospheres. The latter was made by $80 \% \mathrm{RH}$ at $30^{\circ} \mathrm{C}$ because high temperature air contained a larger mount of moisture. Figure 6 and Table 1 contain tensile properties of AL-adsorbed honeycomb-patterned films and flat films. All the films with adsorbed lignin showed higher MOE and tensile strengths than those without lignin under the low moisture content conditions, which suggest effective reinforcement of the cellulose honeycomb frameworks with the isolated lignin.

To compare the effect of lignin adsorption onto honeycomb-patterned film with cellulose 1 polymorphism between the two conditions, their tensile properties in Table 1 were plotted in Figure 7. In the case of no lignin deposition, the observed MOE and tensile strength of the honeycomb-patterned film under the high humidity condition was less than $50 \%$ of that under the normal condition. However, when lignin was adsorbed the MOE remained stable irrespective of humidity. These results seem quite different from the test results of the flat film. That is, the MOE and tensile strength of the flat film with no lignin adsorption were higher under the humid condition than under the normal condition, and those with lignin adsorption were contrarily much less under the humid condition than the normal condition. These tendencies seem only due to experimental variation and no clear difference can be concluded because no reason can be found for both higher MOE of pure cellulose film under higher humidity and a negative effect of adsorbed lignin if we consider water-swelling of cellulose film and water-resistance of lignin.

The above test results imply that swelling of cellulose or cleavage of hydrogen bonds among cellulose molecules allows for easier two dimensional structural deformation of the honeycomb frameworks, likely involving inter-molecule slippage among cellulose chains, which results in a larger apparent axial elongation. ${ }^{[9]}$ The inter-molecule slippage among the tangled cellulose chains that finally result in larger elongation might be restrained by the adsorbed lignin acting as a matrix material. When cellulose chains are arranged randomly and 
densely in the flat film with no such honeycomb pores, it deforms approximately as a solid film without in-plane structural deformation. This mechanical behavior of the flat film may be comparatively stable toward variation in moisture content, as a result lignin adsorption has little potential to improve it. This discussion suggests that the lignin deposition may play a more significant role for structural morphology with pores or internal openings than for solid like dense bodies in mechanical stability under variable moisture content.

The tensile strength of the lignin-adsorbed film showed a tendency a little different from the stability of the MOE; that is, it decreased to be about $75 \%$ of the low moisture conditions under the high moisture conditions. This difference between the test results of MOE and tensile strength of the honeycomb-patterned films is not clearly understood yet, but one of the presumable reasons might be that the adsorbed lignin has a greater effect on the restriction of the swelling of the cellulosic honeycomb frameworks rather than strength reinforcement of them. Because the tensile or bending strength of the honeycomb frameworks are mainly determined by the strength of the bundles of tangled cellulose chains at the weakest cross sections. However, the deformation is the integration of variable local deformation. If lignin would restrict swelling upon water adsorption, MOE at the initial tensile experiment has not been affected.

\section{CONCLUSION}

A woody cell wall-mimicking material was fabricated by the adsorption of lignin onto honeycomb-patterned cellulosic films with cellulose I and II polymorphism. Using such cellulosic films, the mechanical function of lignin was examined by tensile experiments using a manufactured apparatus. It was clearly evident that the lignin (AL) reinforced the cellulosic films. Especially in the higher moisture content atmosphere, lignin adsorption suppressed the decrease in mechanical strength. This effect of lignin adsorption on the mechanical strength of honeycomb-patterned film was not observed for non-porous flat films. This suggests the observed effect in the high moisture content atmosphere is attributed to the honeycomb morphology. Thus, this study has experimentally elucidated the mechanical function of adsorbed isolated lignin on honeycomb-patterned cellulosic films, and illustrated the usefulness of honeycomb-patterned cellulosic films to investigate the functions of woody cell wall components.

Now we are fabricating artificial woody cell wall that further mimics biosynthetic process of cell wall by the deposition of hemicellulose followed by lignin polymerization, to clarify the function of hemicelluloses and native lignin in the cell wall formation.

\section{ACKNOWLEDGEMENT}


This study was financially supported by the Japan Society for the Promotion of Science (\#18380100).

\section{REFERENCES}

1. Nemoto, J.; Uraki, Y.; Kishimoto, T.; Sano, Y.; Funada, R.; Obata, N.; Yabu, H.; Tanaka, M.; Shimomura, M. Production of mesocopically patterned cellulose film. Bioresour. Technol. 2005, 96, 1955-1958.

2. Uraki, Y.; Nemoto, J.; Otsuka, H.; Tamai, Y.; Sugiyama, J.; Kishimoto, T.; Ubukata, M.; Yabu, H.; Tanaka, M.; Shimomura, M. Honeycomb-like architecture produced by living bacteria, Gluconacetobacter xylinus. Carbohydr. Polym. 2007, 69, 1-6.

3. Terashima, N.; Fukushima, K. Heterogeneity in formation of lignin. XI. An autoradiographic study of the heterogeneous formation and structure of pine lignin. Wood. Sci. Technol. 1988, 22, 259-270.

4. Inomata, F.; Takabe, K.; Saiki, H. Cell wall formation of conifer tracheid as revealed by rapid-freeze and substitution method. J. Electron Micro. 1992, 41, 369-374.

5. Ookura, R.; Nishida, J.; Nishikawa, T.; Shimomura, M. Stabilization of micropatterned polymer films as artificial extracellular matrices for tissue engineering. Molecular Crystals and Liquid Crystals Science and Technology Section A -Molecular crystals and Liquid crystals 1999, 337, 461-464.

6. Yabu, H.; Akagi, K.; Shimomura, M. Micropatterning of liquid crystalline polyacetylene derivative by using self-organization processes. Synth. Met. 2009, 159, 762-764.

7. Hirai, Y.; Kojima, M.; Shimomura, M. Simple Fabrication of Honeycomb- and Pincushion-Structured Films Containing Thermoresponsive Polymers and Their Surface Wettability. Chem. Mat. 2009, 21, 1787-1789.

8. Hofstetter, K.; Hellmich, C.; Eberhardsteiner, J. Micromechanical modeling of solid-type and plate-type deformation patterns within softwood materials. A review and an improved approach. Holzforschung 2007, 61, 343-351.

9. Moden, C.S.; Berglund, L.A. Elastic deformation mechanisms of softwoods in radial tension - Cell wall bending or stretching? Holzforshung 2008, 62, 562-568.

10. Nemoto J.; Uraki, Y.; Sano, Y. Preparation of board-like moldings from composites of isolated lignins and waste paper. Mokuzai Gakkaishi 2003, 49, 287-292.

11. Uraki, Y.; Nemoto, J.; Yanaga, K.; Koizumi, A.; Hirai, T. Preparation of board-like moldings from composites of isolated lignins and waste paper II: effect of inorganic salt addition on board performance and evaluation of practical use of MDF. J. Wood Sci. 2005, 51, 589-594. 
12. Fukuhira, Y.; Ito, M.; Kaneko, H.; Sumi, Y.; Tanaka, M.; Yamamoto, S.; Shimomura, M. Prevention of postoperative adhesions by a novel honeycomb-patterned poly(lactide) film in a rat experimental model. J. Biomed. Mater. Res. Part B 2008, 86B, 353-359.

13. Saka, S.; Thomas, R. J.; Gratzl, J. S.; Abson, D. Topochemistry of delignification in Douglas-fir wood with soda, soda-anthraquinone and kraft pulping as determined by SEM-EDXA. Wood Sci. Technol. 1982, 16, 139-53.

14. URL, http://133.50.192.180. The ID and password are required twice when anyone accesses to the URL. ID, edit; Password, journal. 


\section{Figure captions}

Figure 1. Apparatus for fabrication of honeycomb-patterned film based on self-organization of amphiphilic polymer.

Figure 2. Testing apparatus specially designed for tensile tests of small thin specimens. DT shows a linear variable displacement transducer.

Figure 3. Images of agarose medium with a surface of concave type honeycomb (A, B) and honeycomb-patterned film with cellulose I polymorphism (C,D).A, SEM image; B, C and D, 3D-laser microscopic images, where B and D were magnified three times to height;

Figure 4. Time-lapse images of bacterial location using a real-time confocal microscope. Upper images are taking size, and lower images are magnification of upper images. Arrows show the location of bacteria.

Figure 5. SEM images and bromine concentration in the cross section of AL- adsorbed flat bacterial cellulose film (A) and in the surface of AL-adsorbed honeycomb-patterned film with cellulose I polymorphism (B). The lines in the images show the scanning area of bromine by EDX. The arrows show the high concentration area of bromine.

Figure 6. Effects of pore size of honeycomb-patterned film with cellulose II polymorphism on MOE and tensile strength. $\neg$, without lignin deposition; · $\square$.. , with lignin deposition.

Figure 7. MOE and tensile strength of honeycomb-patterned films with cellulose I polymorphism under low moisture conditions (L. C.) and high moisture conditions (H. C.).

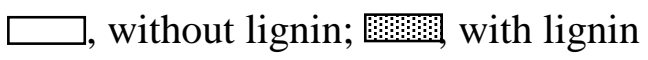




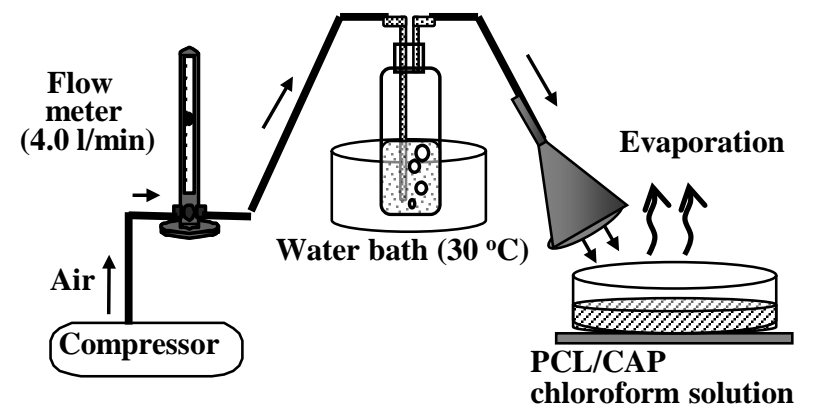

Figure 1. Apparatus for fabrication of honeycombpatterned film based on self-organization of amphiphilic polymer. 


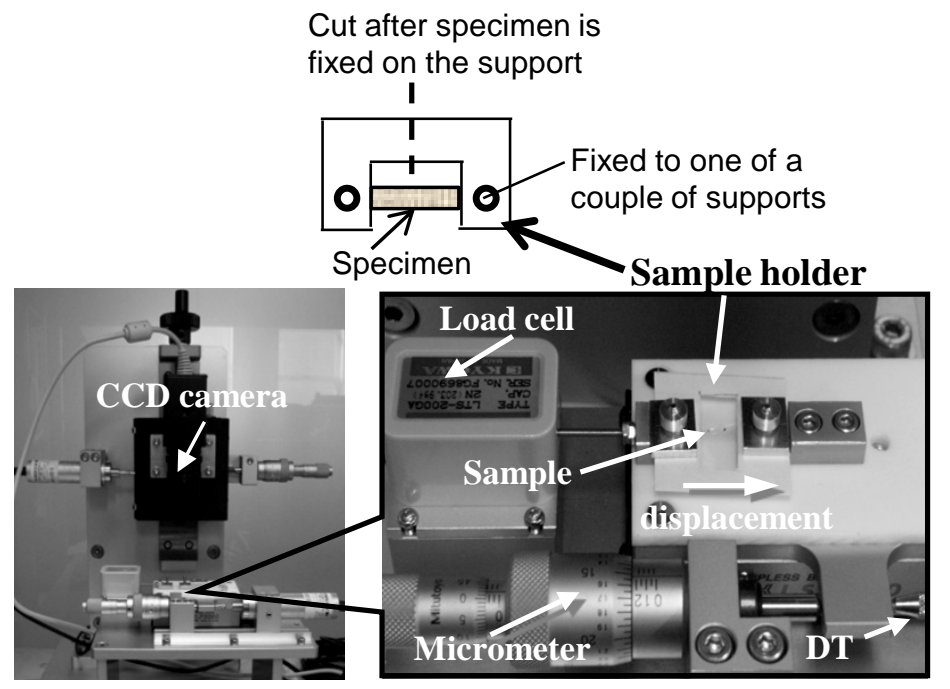

Figure 2. Testing apparatus specially designed for tensile tests of small thin specimens. DT shows a linear variable displacement transducer. 

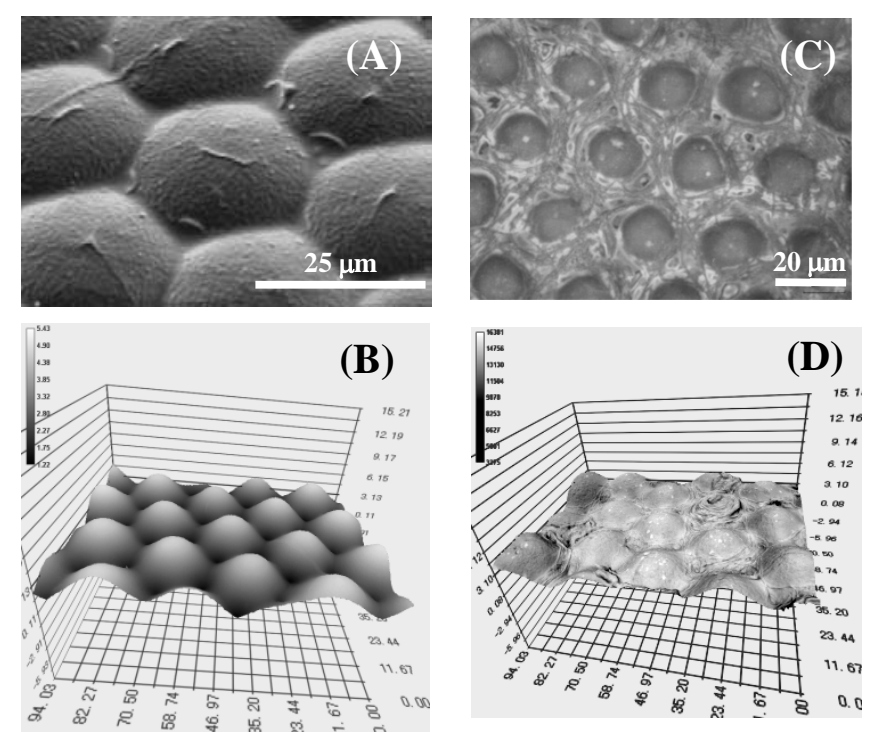

Figure 3. Images of agarose medium with a surface of concave type honeycomb (A, B) and honeycomb-patterned film with cellulose I polymorphism (C,D).A, SEM image; $\mathrm{B}, \mathrm{C}$ and $\mathrm{D}, 3 \mathrm{D}$-laser microscopic images, where $\mathrm{B}$ and $\mathrm{D}$ were magnified three times to height; 


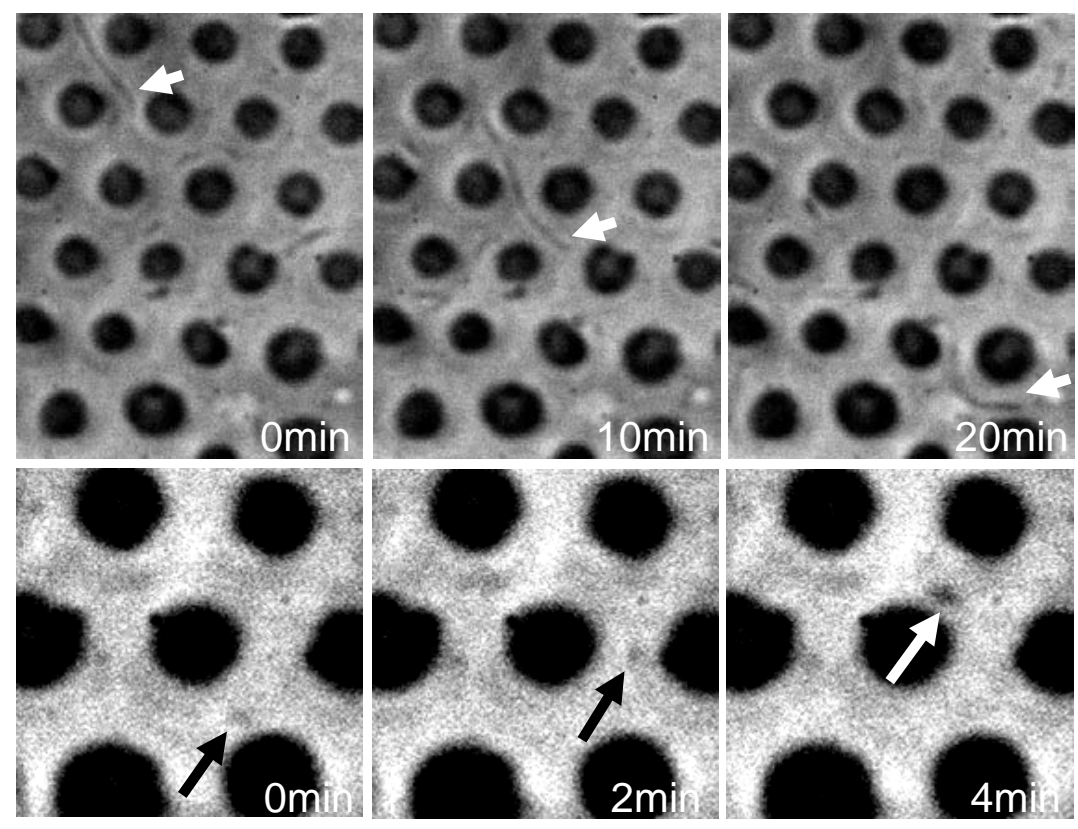

Figure 4. Time-lapse images of bacterial location using an realtime confocal microscope. Upper images are taking size, and lower images are magnification of upper image. Arrows show the location of bacteria. 

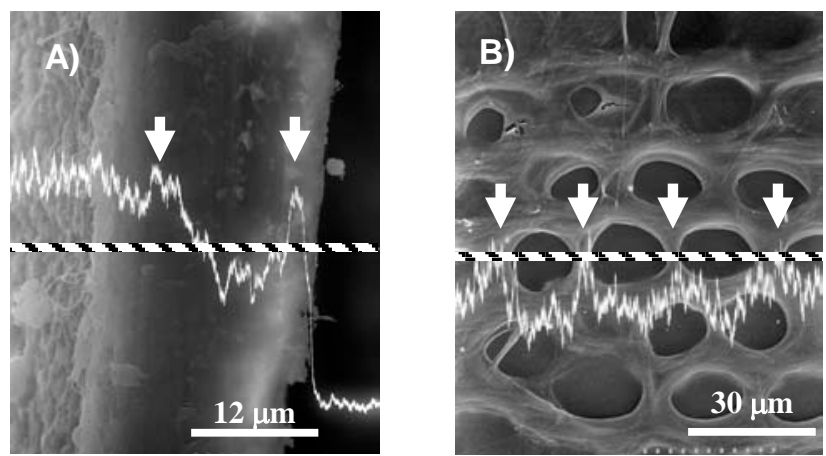

Figure 5. SEM images and bromine concentration in the cross section of AL- adsorbed flat bacterial cellulose film (A) and in the surface of AL-adsorbed honeycomb-patterned film with cellulose I polymorphism (B). The lines in the images show the scanning area of bromine by EDX. The arrows show the high concentration area of bromine. 

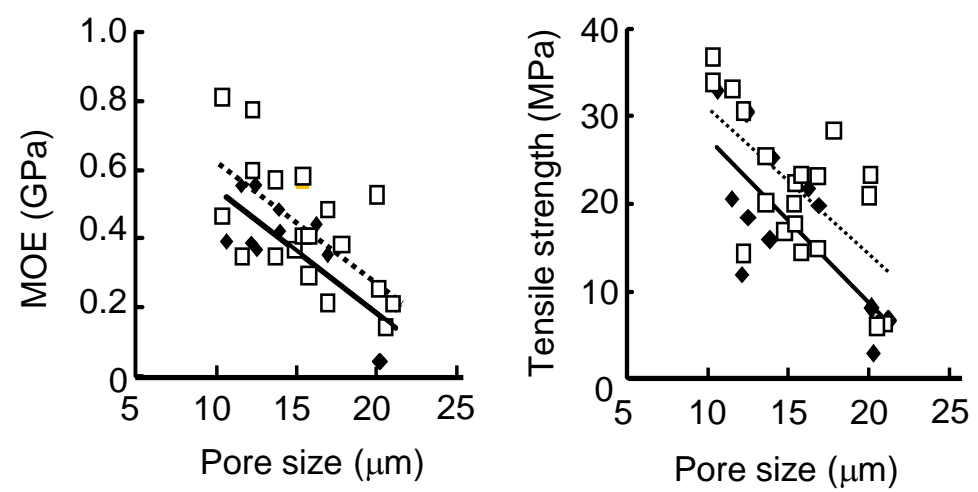

Figure 6. Effects of pore size of honeycomb-patterned film with cellulose II polymorphism on MOE and tensile strength. $\multimap$, without lignin deposition; $\cdot \square \cdot$, with lignin deposition. 

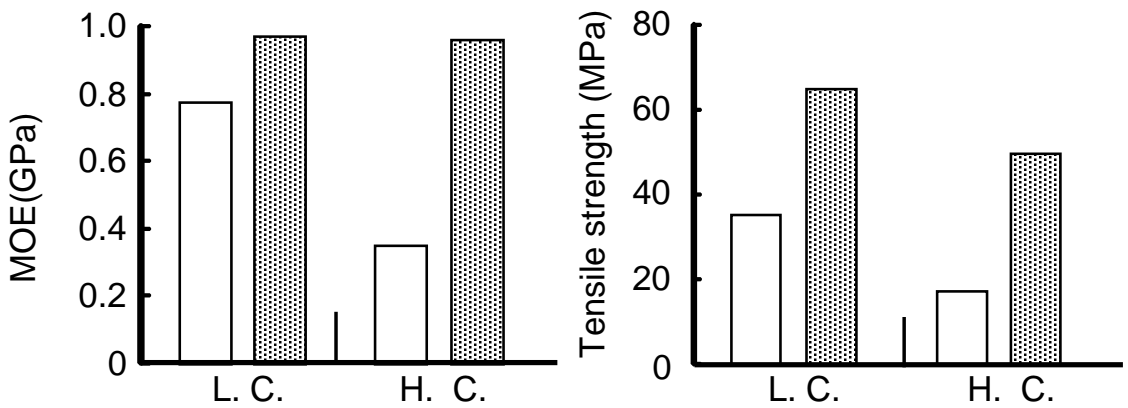

Figure 7. MOE and tensile strength of honeycomb-patterned films with cellulose I polymorphism under low moisture content conditions (L. C.) and high moisture content

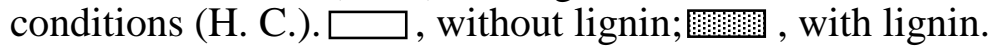


Table 1. Tensile properties of pure cellulosic films and AL-adsorbed films

\begin{tabular}{|c|c|c|c|c|}
\hline & Specimen & $\begin{array}{l}\mathrm{MOE} \\
(\mathrm{GPa})\end{array}$ & $\begin{array}{l}\text { MOR } \\
(\mathrm{MPa})\end{array}$ & $\begin{array}{l}\text { Elongation } \\
\quad(\%)\end{array}$ \\
\hline \multirow{4}{*}{$\begin{array}{l}\text { Normal } \\
\text { conditions } \\
\text { (N. C.) }\end{array}$} & $\begin{array}{l}\text { Flat film } \\
\text { with cellulose I }\end{array}$ & 2.12 & 121 & 10.3 \\
\hline & $\begin{array}{l}\text { AL-adsorbed Flat film } \\
\text { with cellulose I }\end{array}$ & 5.07 & 196 & 5.1 \\
\hline & $\begin{array}{l}\text { Honeycomb-patterned } \\
\text { film with cellulose I }\end{array}$ & 0.77 & 35 & 12.7 \\
\hline & $\begin{array}{l}\text { AL-adsorbed honeycomb- } \\
\text { patterned film with cellulose I }\end{array}$ & 0.95 & 66 & 6.1 \\
\hline \multirow{4}{*}{$\begin{array}{l}\text { High humid } \\
\text { conditions } \\
\text { (H. H. C.) }\end{array}$} & $\begin{array}{l}\text { Flat film } \\
\text { with cellulose I }\end{array}$ & 2.59 & 190 & 9.1 \\
\hline & $\begin{array}{l}\text { AL-adsorbed Flat film } \\
\text { with cellulose I }\end{array}$ & 0.96 & 96 & 11.5 \\
\hline & $\begin{array}{l}\text { Honeycomb-patterned } \\
\text { film with cellulose I }\end{array}$ & 0.35 & 17 & 5.4 \\
\hline & $\begin{array}{l}\text { AL-adsorbed honeycomb- } \\
\text { patterned film with cellulose I }\end{array}$ & 0.96 & 50 & 5.9 \\
\hline \multirow{2}{*}{$\begin{array}{l}\text { Normal } \\
\text { conditions } \\
\text { (N. C.) }\end{array}$} & $\begin{array}{l}\text { Flat film } \\
\text { with cellulose I I }\end{array}$ & 0.56 & 14 & 4.0 \\
\hline & $\begin{array}{l}\text { AL-adsorbed Flat film } \\
\text { with cellulose II }\end{array}$ & 0.65 & 38 & 5.1 \\
\hline
\end{tabular}

Elongation means maximum elongation ratio based on the specimen length before tensile experiment. 